\title{
Naturalness in an Effective Field Theory for Neutron Star Matter
}

\author{
Moisés Razeira*, César A.Z. Vasconcellos*, Bardo E.J. Bodmann ${ }^{\dagger}$, Helio \\ T. Coelho** and Manfred Dillig \\ * Instituto de Física, Universidade Federal do Rio Grande do Sul, CEP 91501-970 Porto Alegre, \\ Rio Grande do Sul, Brazil \\ ${ }^{\dagger}$ Instituto de Física e Matemática, Universidade Federal de Pelotas, CEP 96010-900 Pelotas, Rio \\ Grande do Sul, Brazil \\ **Departamento de Física, Universidade Federal de Pernambuco, CEP 50670-901 Recife, PE \\ Brazil \\ ${ }^{\ddagger}$ Institut für Theoretische Physik III, der Universität Erlangen-Nürnberg, D91058 Erlangen, \\ Germany
}

\begin{abstract}
High density hadronic matter is studied in a generalized relativistic multi-baryon lagrangian density. By comparing the predictions of our model with estimates obtained within a phenomenological naive dimensional analysis based on the naturalness of the coefficients of the theory, we show that naturalness plays a major role in effective field theory and, in combination with experiment, could represent a relevant criterium to select a model among others in the description of global static properties of neutron stars.
\end{abstract}

\section{NATURALNESS OF EFFECTIVE FIELD THEORY}

We consider an interaction lagrangian model for high density nuclear matter[1,2] which contains the isoscalar-scalar meson field $\sigma$, the isoscalar-vector meson field $\omega$ and the isovector-vector meson field $\rho$ coupled to the nucleon field $\psi$

$$
\mathscr{L} \sim C_{l m n p} \frac{1}{m ! n ! p !}\left(\frac{\bar{\psi} \Gamma(\tau / 2) \psi}{f_{\pi}^{2} \Lambda}\right)^{l}\left(\frac{\sigma}{f_{\pi}}\right)^{m}\left(\frac{\omega}{f_{\pi}}\right)^{n}\left(\frac{\rho}{f_{\pi}}\right)^{p}\left(f_{\pi} \Lambda\right)^{2} ;
$$

here $\Gamma$ is a Dirac matrix. After extraction of the dimensional and counting factors, the overall dimensionless coefficients are of order $\mathscr{O}(1)$ if naturalness holds, $C_{l m n p} \sim 1$, resulting in the following expression

$$
\mathscr{L} \sim \frac{1}{m ! n ! p !}\left(\frac{\bar{\psi} \Gamma(\tau / 2) \psi}{f_{\pi}^{2} \Lambda}\right)^{l}\left(\frac{\sigma}{f_{\pi}}\right)^{m}\left(\frac{\omega}{f_{\pi}}\right)^{n}\left(\frac{\rho}{f_{\pi}}\right)^{p}\left(f_{\pi} \Lambda\right)^{2} .
$$

One may compare term by term of the generalized relativistic mean field approach developed in Ref.[3] against the corresponding terms in the natural limit of effective field theory (Eq. 2). The second term of the following expression[2, 4], with $g_{\sigma}$ denoting the scalar coupling constant

$$
-\bar{\psi} M_{N}\left(1+\frac{g_{\sigma} \sigma}{\alpha M_{N}}\right)^{-\alpha} \psi=-\bar{\psi} M_{N} \psi+g_{\sigma} \bar{\psi} \sigma \psi-\frac{1}{2} \frac{\alpha+1}{\alpha M_{N}} g_{\sigma}^{2} \bar{\psi} \sigma^{2} \psi
$$


$-\frac{1}{6} \frac{(\alpha+1)(-\alpha-2)}{\left(\alpha M_{N}\right)^{2}} g_{\sigma}^{3} \bar{\psi} \sigma^{3} \psi-\frac{1}{24} \frac{(\alpha+1)(-\alpha-2)(-\alpha-3)}{\left(\alpha M_{N}\right)^{3}} g_{\sigma}^{4} \bar{\psi} \sigma^{4} \psi+\bar{\psi} \mathscr{O}\left(\sigma^{5}\right) \psi$,

shall be compared with

$$
\mathscr{L}_{\sigma \psi} \sim C_{1100} \frac{1}{1 ! 0 ! 0 !}\left(\frac{\bar{\psi} \psi}{f_{\pi}^{2} M_{N}}\right)^{1}\left(\frac{\sigma}{f_{\pi}}\right)^{1}\left(f_{\pi} M_{N}\right)^{2}
$$

where the following values are understood: $m=1, n=p=0$ and $\Lambda=M_{N}$, giving for the corresponding coupling constant

$$
\sim C_{1100}\left(\frac{M_{N}}{f_{\pi}}\right) \bar{\psi} \sigma \psi=10.09677 C_{1100} \bar{\psi} \sigma \psi, C_{1100} \sim \frac{g_{\sigma}}{10.09677} \sim 1 .
$$

This example indicates the 1100 term has a natural coupling with $C_{1100} \sim 1$. The same result holds for the other terms.

In the description of properties of nuclear matter we consider the lagrangian density[3]

$$
\begin{aligned}
\mathscr{L} & =\sum_{B=p, n, \Lambda, \Sigma^{-}, \Sigma^{0}, \Sigma^{+}, \Xi^{-}, \Xi^{0}}\left\{\overline { \psi } _ { B } \left(\left[1+\frac{\left(g_{\sigma} \sigma+g_{\delta} \vec{\tau}_{B} \cdot \vec{\delta}\right)}{\alpha M_{B}}\right]^{\alpha} \gamma_{\mu}\left(i \partial^{\mu}-g_{\omega} \omega^{\mu}-\frac{1}{2} g_{\rho} \tau_{B} \cdot \rho^{\mu}\right)\right.\right. \\
& \left.-\left(1-\frac{g_{\sigma} \sigma}{M_{B}}\right)^{\eta} M_{B}\right) \psi_{B}+\frac{1}{2}\left(1+\frac{g_{\sigma} \sigma}{\beta M_{B}}\right)^{2 \beta}\left(\frac{1}{2} m_{\omega}^{2} \omega_{\mu} \omega^{\mu}-\frac{1}{4} \omega_{\mu \nu} \omega^{\mu v}\right) \\
& \left.+\frac{1}{2}\left(1+\frac{g_{\sigma} \sigma}{\gamma M_{B}}\right)^{2 \gamma}\left(\frac{1}{2} m_{\rho}^{2} \rho_{\mu} \cdot \rho^{\mu}-\frac{1}{4} \rho_{\mu v} \cdot \rho^{\mu v}\right)\right\}+\left(\frac{1}{2} \partial_{\mu} \sigma \partial^{\mu} \sigma-\frac{1}{2} m_{\sigma^{2} \sigma^{2}}\right) \\
& +\sum_{\lambda} \bar{\psi}_{\lambda}\left[i \gamma_{\mu} \partial^{\mu}-m_{\lambda}\right] \psi_{\lambda}
\end{aligned}
$$

where $\omega_{\mu v}=\partial_{\mu} \omega_{v}-\partial_{v} \omega_{\mu}$ and $\rho_{\mu v}=\partial_{\mu} \rho_{v}-\partial_{v} \rho_{\mu}$. The isospin extension is necessary due to the large isospin-asymmetry in neutron matter. This lagrangian density describes a baryon octet $\left(B=p, n, \Lambda, \Sigma^{-}, \Sigma^{0}, \Sigma^{+}, \Xi^{-}, \Xi^{0}\right)$ coupled to four mesons $(\sigma, \omega, \rho$ and $\delta$ ) and two leptons $(e, \mu)$ and contains a gradient coupling involving the baryons and the scalar-isoscalar and scalar-isovector meson fields. The different steps of our formulation may be synthesized as follows. We first introduce a re-scaling of the baryons and the $\omega$ and $\rho$ meson fields: $\phi \rightarrow\left[1+\frac{\left(g_{\sigma} \sigma+g_{\delta} \vec{\tau}_{B} \cdot \vec{\delta}\right)}{\alpha M_{B}}\right]^{-\alpha / 2} \phi$ with $\phi=\psi_{B}, \omega^{\mu}, \rho^{\mu}$. Further we combine the resulting expressions with the previous ones and define new coupling constants: $g_{\alpha \sigma B}^{\star} \equiv m_{\alpha B}^{\star} g_{\sigma} ; g_{\beta \omega B}^{\star} \equiv m_{\beta B}^{\star} g_{\omega} ; g_{\gamma \rho B}^{\star} \equiv m_{\gamma B}^{\star} g_{\rho} ; g_{\alpha \delta B}^{\star}=m_{\alpha B}^{\star} g_{\delta}$ with $m_{\kappa B}^{\star} \equiv$ $\left(1+\left(g_{\sigma} \sigma+g_{\delta} \tau_{3 B} \delta_{3}\right) / \kappa M\right)^{-\kappa}$, with $(\kappa=\alpha, \beta, \gamma)$ where $\tau_{3 B}$ is the third component of the baryons isospin vectors $\vec{\tau}_{B}$ and $\sigma$ and $\delta_{3}$ are, respectively, the mean field components of the $\sigma$ and $\delta$ mesons. The effective baryon mass is defined as

$$
M_{N}^{*}=M_{N}\left(1-\frac{g_{\sigma} \sigma}{M_{N}}\right)^{\eta}\left[1+\frac{\left.\left(g_{\sigma} \sigma+g_{\delta} \vec{\tau}_{N} \cdot \vec{\delta}\right)\right)}{\alpha M_{N}}\right]^{-\alpha} .
$$

Up to first order in $\sigma$ and in the limit $\alpha=1, \eta=0$ and $g_{\delta}=0$, the re-scaled Lagrangian gives for the effective nucleon mass the well known Yukawa minimal coupling $M_{N}^{*} \simeq$ $M_{N}-g_{\sigma} \sigma$. In our calculations $g_{\delta}=0$. 

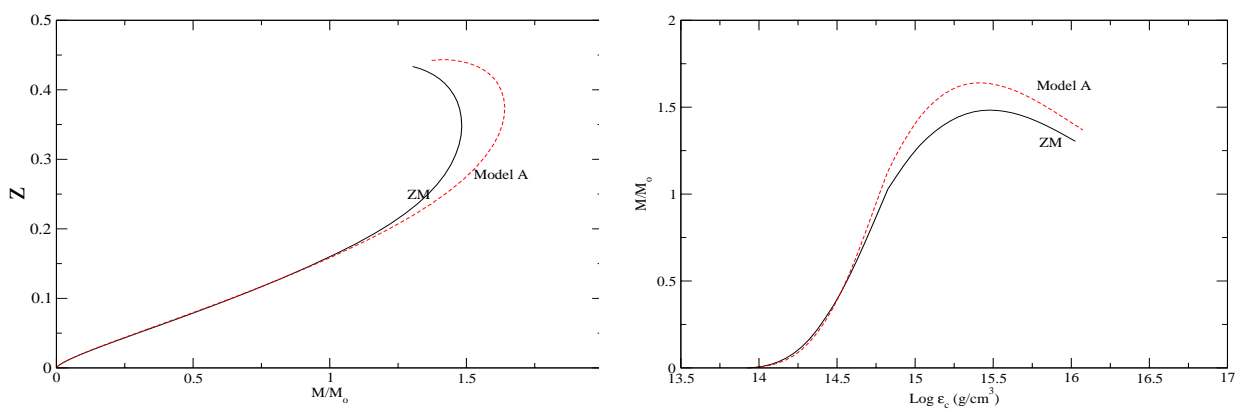

FIGURE 1. On the left panel, predictions of our model for the gravitational red-shift of neutron stars. On the right panel, maximum mass of neutron matter as predicted by our model as a function of the central density. Compared are the results of our model (A) with the ZM model.

\section{RESULTS AND CONCLUSIONS}

Our results indicate that, for our modelling of nuclear matter and neutron stars, naturalness holds. In particular, our predictions indicate, in comparison with recent experimental results for the mass and red-shift of neutron stars[7], that naturalness puts stringent constraints on observables in effective models, and therefore might be an useful indicator to discriminate current relativistic models of nuclear and neutron star matter.

\section{REFERENCES}

1. B. D. Serot and J. D. Walecka, Int. J. Mod. Phys. E6 515 (1997).

2. C. Maekawa et al. (contribution to this volume).

3. S.S. Rocha et al. (contribution to this volume).

4. K. Saito, K. Tsushima, A.W. Thomas, Phys. Lett. B406 (1997) 287.

5. N.K. Gledenning, Astrophys. J. 293 (1985) 470.

6. J. Zimanyi, S.A. Moszkowski, Phys. Rev. C42 (1990) 1416.

7. J. Cottam, F. Paerels, M. Mendez, Nature 420 (2002) 51. 
Copyright of AIP Conference Proceedings is the property of American Institute of Physics and its content may not be copied or emailed to multiple sites or posted to a listserv without the copyright holder's express written permission. However, users may print, download, or email articles for individual use. 